\title{
Which test for crossing survival curves? A user's guideline
}

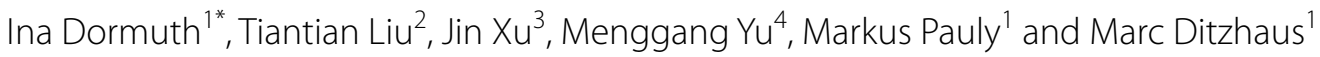

\begin{abstract}
Background: The exchange of knowledge between statisticians developing new methodology and clinicians, reviewers or authors applying them is fundamental. This is specifically true for clinical trials with time-to-event endpoints. Thereby, one of the most commonly arising questions is that of equal survival distributions in two-armed trial. The log-rank test is still the gold-standard to infer this question. However, in case of non-proportional hazards, its power can become poor and multiple extensions have been developed to overcome this issue. We aim to facilitate the choice of a test for the detection of survival differences in the case of crossing hazards.

Methods: We restricted the review to the most recent two-armed clinical oncology trials with crossing survival curves. Each data set was reconstructed using a state-of-the-art reconstruction algorithm. To ensure reproduction quality, only publications with published number at risk at multiple time points, sufficient printing quality and a noninformative censoring pattern were included. This article depicts the $p$-values of the log-rank and Peto-Peto test as references and compares them with nine different tests developed for detection of survival differences in the presence of non-proportional or crossing hazards.

Results: We reviewed 1400 recent phase III clinical oncology trials and selected fifteen studies that met our eligibility criteria for data reconstruction. After including further three individual patient data sets, for nine out of eighteen studies significant differences in survival were found using the investigated tests. An important point that reviewers should pay attention to is that $28 \%$ of the studies with published survival curves did not report the number at risk. This makes reconstruction and plausibility checks almost impossible.

Conclusions: The evaluation shows that inference methods constructed to detect differences in survival in presence of non-proportional hazards are beneficial and help to provide guidance in choosing a sensible alternative to the standard log-rank test.
\end{abstract}

Keywords: Survival analysis, Time-to-event outcome, Crossing, Non-proportional hazards, Oncology, Log-rank test, Restricted-mean survival

\section{Background}

Time-to-event studies are the paramount studies in clinical practice. Typical examples are two-armed trials providing a reliable comparison of the efficacy and safety of two treatments. Statistical methods that infer a potential

*Correspondence: Ina.dormuth@tu-dortmund.de

1 TU Dortmund University, Joseph-von-Fraunhofer-Straße 2-4,

44221 Dortmund, Germany

Full list of author information is available at the end of the article difference in survival are of fundamental importance [1]. Among methods designed to compare the overall survival of two groups, the log-rank test (LR) is still the most used [2]. Beyond a certain resistance to statistical innovations [3], there is also a theoretical reason: The LR is optimal in case of proportional hazards $(\mathrm{PH})$ [4]. In other words, if the hazard functions of the two groups are proportional, the LR is the most powerful method to detect differences between them. However, this changes completely for other kinds of hazard patterns, in particular for crossing 
hazards and the rejection rates of the LR drop significantly. The alarming observation of Kristiansen [5], who reviewed 175 studies in five renowned journals, is that the LR was applied in $70 \%$ of the cases despite crossing survival curves. These crossings can occur e.g. in oncology when comparing tumor dissection versus radiation strategies due to different time-dependent effects.

Consequently, several methods have been and are still proposed to tackle non-PH situations. However, due to the speed of research and the number of new methods, the exchange of knowledge is a challenge. Therefore, Ananthakrishnan et al. [6] recently provided a critical review on methods in the presence of possible non-PHs and their limitations and advantages. While they give detailed information regarding the assumptions and the context, they do not provide any numerical evaluation of the methods. We include here state of the art tests with the aim of providing biostatisticians, physicians and reviewers with a condensed overview of suitable methods for non-PH settings that are implemented in the open statistical software $\mathrm{R}$. These methods not only show good results in various simulation studies but also on real data.

\section{Methods}

There are several papers that develop alternatives to the LR in case of non-PH or even crossing hazards. Treating them all would go far beyond the scope of this work. Hence, we focused our comparisons on standard methods that performed well in other simulation studies and more recent ones that were not yet included in extensive evaluations. Here, all analyses are conducted using the free and open-source software R [7] (except for the test introduced by Royston [8]).

Fortunately, the paper by Li et al. [9] already provides a review on methods for crossing hazards up to 2014 . Based on extensive simulation studies they recommend two procedures: First, Neyman's smooth test proposed by Kraus [10]. This test is not considered further since the corresponding R package was removed recently. Second, a two-stage procedure (2ST) that is based on the LR and a crossing-hazards test is proposed (see the Supplement for more details.). The test is described by Qiu and Sheng [11] and implemented in the R package TSHRC [12].

Further methods have been developed since 2014. We have included the most relevant ones into our study. For example, Gorfine et al. [13] presented two omnibus permutation tests based on a sample space partition, which showed promising results in non-PH situations. These are either based on test statistics of Pearson's chi square (KONP chi) or likelihood-ratio type (KONP llr) and are available in the $\mathrm{R}$ package KONPsurv [14]. They compared their new approach with the well-established test of Yang and Prentice [15], which belongs to the class of weighted log-rank tests and employs adaptive weights. Since Gorfine et al. [13] could show in simulations that their new tests are more powerful in the studied nonPH settings, the Yang and Prentice test is not included in our comparison. Another idea starts with the class of weighted LR. This class is long known and includes the LR as well as the common Peto-Peto test (PP). Recently, a flexible combination of several weighted LRs into one test procedure was proposed [16-18]. It is based upon a combination of alternatives and carried out as a permutation procedure. Recently, it has been implemented in the R package mdir.log-rank [19]. The multiple-direction log-rank test (mdir) combines several weighted log-rank tests into one joint Wald-type statistic, which can be interpreted as a projection on a large alternative space spanned by pre-chosen weights. The latter ensures that mdir has not only a reasonable power in the directions of the chosen weights (e.g. for PHs or a specific crossing curve situation) but also in the directions of any linear combination of the pre-chosen weights. Moreover, the weights are allowed to be data-dependent. Another approach that combines multiple weighted log-rank tests is the MaxCombo test (MaxCombo). Different to mdir, the final test statistic is the maximum over standardized weighted LR tests [20]. We used the same list of weights as proposed in the description of the nphsim package [21]. We refer to the supplement for specific as well as technical details on all methods. Besides HR, the restricted mean survival time (RMST) can be used to quantify the difference between two survival curves [22]. It describes the mean event-free survival time up to a pre-defined time point $\tau$. Hypothesis tests constructed using the RMST examine whether the RMST difference between groups is zero. This test is also valid to test equality of two survival functions, since equal survival functions imply equal RMST. Unfortunately, it is possible to observe situations where the RMSTs are equal but the survival functions are not. This has to be kept in mind while using RMST-based tests. We consider three RMST-based proposals: The first two utilize the groupwise RMST differences as test statistic and either calculate $p$-values based on resampling (RMST1) or obtained using asymptotic theory (RMST2) [23, 24]. The former is provided by the R package surv2sample [25] while the latter can be computed with the function rmst 2 in survRM2 [26]. Eventually, Royston and Parmar [27, 28] propagate a test combining a Cox test and a permutation-based RMST test (coxRMST). The test by Royston and Parmar is only available in STATA using the stctest function. Finally, we consider a test based on an integrated $L_{1}$-distance of the two Kaplan-Meier curves as test statistic. It can be interpreted as the area between curves (ABC) and was introduced in Liu et al. [29]. It has not been 
implemented in $\mathrm{R}$ yet and was thus coded by ourselves according to the author's descriptions. The code can be found in the supplements.

A detailed description of all eleven tests and corresponding test statistics can be found in the Supplement. Furthermore, a simple example in $\mathrm{R}$ is given in the Supplement. Below we will compare them based upon different studies. To this end, we reconstruct data from published Kaplan-Meier curves using the algorithm developed by Guyot et al. [30] and deriving the data from the curves with the freely available Webplotdigitizer [31].

\section{Results}

\section{Eligibility screening and data extraction}

Our study was motivated by the work of Matabuena and Padilla [32] which includes three oncology studies with crossing Kaplan Meier (KM) curves. We subsequently performed a PubMed screening of recent oncology studies with similar patterns. To ensure these patterns, the search matched ((Phase 3) OR (phase III)) OR (KaplanMeier) OR (Kaplan Meier)) for Cancer and Humans were used. To categorize them, multiple criteria listed in Fig. 1 were defined to identify relevant studies on PubMed. 1400 of the most recent papers (status from Oct 5,2020 ) on clinical oncology were searched for crossing survival curves with published number at risk at multiple time points. More details can be found in eTable 1 in the online Supplement. The executed LR test had to be non-significant and the two arms should only cross one or two times. To ensure a good reconstructibility, a sufficient number of events and high quality of the curves as well as non-informative censoring over time were required. In the end, the reconstruction algorithm of Guyot et al. [30] was applied to fifteen publications that met these requirements and the three studies discussed in the paper of Matabuena and Padilla [32]. Beyond insufficient information (e.g., almost $30 \%$ of the publications did not report the number at risks) another reason for the final small number of publications can be publication bias since non-significant results are less often reported.

\section{Data reconstruction}

The individual patient data from the three studies found in Matabuena and Padilla [32] and the fifteen other studies under consideration [33-50] were reconstructed using the algorithm introduced by Guyot et al. [30]. To assess the quality of reconstruction, the reported key statistics (median survival and HR with confidence interval) published in each paper were recalculated and compared to the original values (see Table 1).

\section{Comparison of tests for proportional hazards and crossing hazards}

The reconstructed individual patient data were then used to compare the different testing approaches. For all resampling-based methods, the number of iterations was set to 5000 and for all RMST procedures the parameter $\tau$ was set to $90 \%$ of the minimum of the largest censored or uncensored time among the arms [51]. The results are listed in Table 2.

It can be observed that the LR test never succeeds to reject the null hypothesis of equal survival in both groups at the $5 \%$ level. This leads to the exact same conclusion as in the eighteen published studies. The PP is designed to find early differences [52]. It succeeds in revealing an inequality in survival for four of the eighteen studies under consideration [33, 40, 45, 47]. Let us next consider the three RMST tests. These do not rely on the assumption of $\mathrm{PHs}$ but are also not specifically designed to detect crossings [53]. The resampling-based (RMST1) and the distribution-based version (RMST2) reject the null hypothesis in three cases [33, 34, 40], while the combined test (coxRMST) rejects the null hypothesis in five cases [33, 39, 40, 45, 47]. These findings support the analyses of Royston et al. [54]. The six remaining tests are all omnibus tests with different properties. The two tests by Gorfine et al. [13]. (KONP chi and KONP llr) find differences in survival in the same six cases $[33,34,41$, 42, 45, 47]. The omnibus test by Ditzhaus and Friedrich [17] (mdir) can reject the null hypothesis in eight out of eighteen cases [33, 37, 39-41, 45, 47]. The two-stage procedure (2ST) detects differences in five out of eighteen data sets [33, 40, 41, 45, 47]. The ABC has significant results for the same five studies as the two-stage test [33, $40,41,45,47]$. The MaxCombo test leads to $p$-values smaller than 0.05 for seven of the eighteen data sets [34, $39-42,45,47]$. In these specific data examples, the test by Ditzhaus and Friedrich [14] is the test that detects the most differences. These results are consistent with those of Li et al. [9], Gorfine et al. [13] and Royston and Parmar [28] who also indicated that omnibus tests have greater power when deviating from the proportional hazards assumption. Evaluation of the methods' performance

\begin{tabular}{|c|c|c|c|c|c|}
\hline $\begin{array}{l}\text { Papers viewed on } \\
\text { PubMed } \\
N=1400\end{array}$ & $\begin{array}{l}\text { Papers with survival } \\
\text { curves published } \\
n=689\end{array}$ & $\begin{array}{l}\text { Papers with two group } \\
\text { comparsion } \\
n=507\end{array}$ & $\begin{array}{c}\text { Papers with one or two } \\
\text { crossings } \\
n=169\end{array}$ & $\begin{array}{c}\text { Papers with non- } \\
\text { - significant log-rank test } \\
n=132\end{array}$ & $\begin{array}{l}\text { Papers with sufficient } \\
\text { quality for reproduction } \\
n=15\end{array}$ \\
\hline
\end{tabular}

Fig. 1 Flow chart of papers under consideration 
Table 1 Assessment of data reconstruction quality

\begin{tabular}{llll}
\hline Publication & MS G1 & MS G2 & HR [Cl] \\
\hline Bang et al. (2020) [37] & $5.80(5.88)$ & $4.30(4.44)$ & $0.83[0.53,1.31](0.82[0.52,1.29])$ \\
Becker et al. (2020) [39] & not defined & $6.00(6.21)$ & $5.50(5.51)$ \\
Bellmunt et al. (2017) [45] & $3.30(3.24)$ & $2.10(2.08)$ & $0.98[0.81,1.19](0.93[0.77,1.13])$ \\
Cortes et al. (2019) [46] & $4.90(4.94)$ & $4.70(4.72)$ & $0.63(0.62)$ \\
Ferris et al. (2016) [41] & $2.00(2.02)$ & $2.30(2.29)$ & $0.89[0.70,1.13](0.89[0.70,1.14])$ \\
Fradet et al. (2019) [47] & $3.30(3.35)$ & $2.10(2.16)$ & $0.96[0.79,1.16](0.92[0.77,1.11])$ \\
Godfrey et al. (2018) [36] & - & - & $1.40[0.54,3.61](1.40[0.53,3.69])$ \\
Golan et al. (2019) [38] & $18.90(18.90)$ & $18.10(18.10)$ & $0.91[0.56,1.46](0.88[0.55,1.42])$ \\
Hammel et al. (2019) [35] & $21.20(21.36)$ & $6.00(5.93)$ & $0.72[0.41,1.27](0.72[0.42,1.24])$ \\
Jones et al. (2020) [34] & $26.00(26.00)$ & $20.0(18.80)$ & $0.59[0.34,1.05](0.58[0.33,1.02])$ \\
Jones et al. (2018) [33] & $15.10(15.08)$ & $8.10(8.02)$ & $0.72[0.45,1.17](0.71[0.44,1.15])$ \\
Kotani et al. (2019) [48] & $8.60(8.62)$ & $8.00(8.02)$ & $0.74[0.48,1.14](0.72[0.47,1.11])$ \\
Kreuzer et al. (2020) [50] & $19.40(19.40)$ & $20.90(21.30)$ & $1.22[0.60,2.47](1.26[0.62,2.56])$ \\
Lu et al. (2018) [40] & $4.63(4.68)$ & $4.23(4.33)$ & $0.78[0.60,1.00](0.74[0.55,1.01])$ \\
Malone et al. (2020) [49] & - & - & $0.66[0.41,1.07](0.68[0.42,1.10])$ \\
Motzer et al. (2015) [42] & $4.60(4.46)$ & $4.40(4.07)$ & $0.88[0.75,1.03](0.87[0.98,1.34])$ \\
Mukai et al. (2019) [44] & $27.90(27.90)$ & $16.60(16.60)$ & $0.55[0.23,1.29](0.55[0.23,1.29])$ \\
Toxopeus et al. (2018) [43] & - & - & $1.02[0.75,1.39](1.01[0.75,1.39])$ \\
\hline
\end{tabular}

Quality of data reconstruction regarding the published median survival (MS) in group 1 and 2 (G1 and G2), the hazard ratio (HR) with 95\% confidence intervals (CI). For each study the published statistics are given with the corresponding statistics of the reconstructed data in parentheses. Three studies did not report MS (-) and two did not provide confidence intervals

Table $2 P$-values of the different tests applied to the reconstructed individual patient data of each publication

\begin{tabular}{llllllllllll}
\hline Publication & LR & PP & RMST1 & RMST2 & coxRMST & KONP_chi & KONP_IIr & Mdir & 2ST & ABC & MaxCombo \\
\hline Bang et al. (2020) [37] & 0.37 & 0.07 & 0.11 & 0.12 & 0.11 & 0.14 & 0.15 & $\mathbf{0 . 0 3}$ & 0.06 & 0.13 & 0.1 \\
Becker et al. (2020) [39] & 0.09 & 0.47 & 0.22 & 0.22 & $\mathbf{0 . 0 2}$ & 0.14 & 0.09 & $\mathbf{0 . 0 2}$ & 0.27 & 0.15 & $\mathbf{0 . 0 4}$ \\
Bellmunt et al. (2017) [45] & 0.49 & $\mathbf{0 . 0 3}$ & 0.38 & 0.38 & $\mathbf{0 . 0 0 3}$ & $<\mathbf{0 . 0 0 1}$ & $<\mathbf{0 . 0 0 1}$ & $<\mathbf{0 . 0 0 1}$ & $\mathbf{0 . 0 3}$ & $\mathbf{0 . 0 0 2}$ & $<\mathbf{0 . 0 0 1}$ \\
Cortes et al. (2019) [46] & 0.19 & 0.24 & 0.23 & 0.24 & 0.28 & 0.40 & 0.36 & 0.41 & 0.87 & 0.29 & 0.56 \\
Ferris et al. (2016) [41] & 0.33 & 0.84 & 0.23 & 0.23 & 0.25 & $\mathbf{0 . 0 1}$ & $\mathbf{0 . 0 0 9}$ & $\mathbf{0 . 0 2}$ & $\mathbf{0 . 0 4}$ & $\mathbf{0 . 0 3}$ & $<\mathbf{0 . 0 0 1}$ \\
Fradet et al.(2019) [47] & 0.40 & $\mathbf{0 . 0 2}$ & $\mathbf{0 . 0 4}$ & $\mathbf{0 . 0 4}$ & $\mathbf{0 . 0 0 9}$ & $<\mathbf{0 . 0 0 1}$ & $<\mathbf{0 . 0 0 1}$ & $<\mathbf{0 . 0 0 1}$ & $\mathbf{0 . 0 3}$ & $\mathbf{0 . 0 0 1}$ & $<\mathbf{0 . 0 0 1}$ \\
Godfrey et al. (2018) [36] & 0.49 & 0.48 & 0.58 & 0.59 & 0.63 & 0.18 & 0.20 & 0.75 & 0.90 & 0.44 & 0.74 \\
Golan et al. (2019) [38] & 0.61 & 0.78 & 9.74 & 0.75 & 0.50 & 0.58 & 0.59 & 0.61 & 0.22 & 0.61 & 0.66 \\
Hammel et al. (2019) [35] & 0.22 & 0.35 & 0.62 & 0.62 & 0.33 & 0.19 & 0.19 & 0.16 & 0.27 & 0.38 & 0.09 \\
Jones et al. (2020) [34] & 0.05 & 0.11 & 0.14 & 0.14 & 0.07 & $\mathbf{0 . 0 2}$ & $\mathbf{0 . 0 2}$ & 0.12 & 0.41 & 0.11 & $\mathbf{0 . 0 4}$ \\
Jones et al. (2018) [33] & 0.17 & $\mathbf{0 . 0 3}$ & $\mathbf{0 . 0 2}$ & $\mathbf{0 . 0 2}$ & $\mathbf{0 . 0 5}$ & $\mathbf{0 . 0 3}$ & $\mathbf{0 . 0 4}$ & $\mathbf{0 . 0 0 9}$ & $\mathbf{0 . 0 5}$ & $\mathbf{0 . 0 3}$ & $0.05^{\mathrm{a}}$ \\
Kotani et al. (2019) [48] & 0.14 & 0.24 & 0.38 & 0.38 & 0.20 & 0.48 & 0.48 & 0.28 & 0.45 & 0.45 & 0.17 \\
Kreuzer et al. (2020) [50] & 0.53 & 0.25 & 0.07 & 0.08 & 0.17 & 0.28 & 0.28 & 0.10 & 0.10 & 0.07 & 0.27 \\
Lu et al. (2018) [40] & 0.06 & $\mathbf{0 . 0 0 7}$ & $\mathbf{0 . 0 2}$ & $\mathbf{0 . 0 2}$ & $\mathbf{0 . 0 2}$ & 0.07 & 0.07 & $\mathbf{0 . 0 0 7}$ & $\mathbf{0 . 0 4}$ & $\mathbf{0 . 0 1}$ & $\mathbf{0 . 0 1}$ \\
Malone et al. (2020) [49] & 0.11 & 0.12 & 0.13 & 0.13 & 0.17 & 0.08 & 0.09 & 0.28 & 0.57 & 0.12 & 0.22 \\
Motzer et al. (2015) [42] & 0.07 & 0.51 & 0.13 & 0.13 & 0.11 & $\mathbf{0 . 0 3}$ & $\mathbf{0 . 0 3}$ & $\mathbf{0 . 0 1}$ & 0.26 & 0.08 & $<\mathbf{0 . 0 0 1}$ \\
Mukai et al. (2019) [44] & 0.17 & 0.22 & 0.15 & 0.17 & 0.26 & 0.22 & 0.25 & 0.33 & 0.53 & 0.16 & 0.44 \\
Toxopeus et al. (2018) [43] & 0.91 & 0.84 & 0.75 & 0.75 & 0.35 & 0.37 & 0.36 & 0.15 & 0.11 & 0.56 & 0.34 \\
\hline
\end{tabular}

a Only 0.05 due to rounding down

Bold values indicate $p$-values smaller than the $5 \%$ type-I error level 
under PHs reveals that almost all of the approaches reject the null hypothesis when the LR does (for details see the Supplement). In future simulation studies, the performance of the tests and their extensions to multi-arm settings will be further evaluated [13,55-57].

\section{Discussion}

To assess efficacy of two treatments the LR is generally regarded as the gold standard. The LR is optimal in terms of power under the $\mathrm{PH}$ assumption but can lose sufficient power in non-PH situations. The results of our PubMed analysis, however, show that there are many situations, where the LR is used in case of non-PH. At the same time, several alternatives are presented, which succeed to detect differences where the LR fails. The majority of these tests are available in statistical software (R). Hence, their execution is almost as user-friendly as calculating the LR. To furtherfacilitate their application, we provide minimal examples on how to use the implemented $R$ functions in the supplement.

To exemplify the different implications, we reconstructed individual patient data from eighteen recent oncology trials that met the eligibility criteria of our analysis. In particular, high quality KM plots with sufficient information were necessary for the reconstruction algorithm. Based on these eighteen studies we compared the test decisions of eleven different testing procedures. It turns out, that the LR alternatives can exhibit power to identify differences between groups. Omnibus approaches, which have high power against several alternatives (such as $\mathrm{PH}$ and crossings in case of the mdir test), turned out to be particularly suitable for this purpose (see the Supplement for additional information regarding $\mathrm{PH}$ performance).

\section{Limitations}

One of the main limitations of this kind of study is the dependence on the selection of data sets. To make a clear statement regarding the quality of the individual procedures in a direct comparison, extensive simulation studies are necessary. These are part of our own ongoing research. Nevertheless, it can be said that the LR cannot reject the null hypothesis in real situations involving non-proportional hazards included in this paper, while various omnibus tests are able to do so. Furthermore, the data used here are reconstructed individual patient data and thus does not have the same quality as the original data. While many properties of the data such as non-proportionality are conserved, the biggest reconstruction issue is the assumption of uniformly distributed censoring times. However, the assessment of the reconstruction quality turned out to be very satisfying.

\section{Recommendations for reviewers}

Regarding the insights of our investigation, attention in the reviewing process of study reports should be paid to

(1) the appropriate choice of the statistical method. Especially when the $\mathrm{PH}$ assumption cannot be justified in advance, e.g. by a preliminary study, alternatives to the LR should be considered. Due to multiplicity issues, we do not advocate the common practice of pre-testing the $\mathrm{PH}$ assumption. Instead, we suggest directly applying a procedure which can detect survival curve differences in $\mathrm{PH}$ as well as non-PH settings, such as the methods presented in this paper.

(2) the quality of the data presentation and the report of all relevant information. This includes, in particular, the table of the number at risks at multiple time points, which was not reported in almost 30\% of the reviewed publications. These tables and all relevant information can be easily accessed through each common statistical software and should be provided in every study report. They are mandatory for a reliable assessment of the results and, moreover, facilitate a secondary analysis, e.g. for metaanalysis studies, by reconstructing the original data in a reasonable quality [25].

\section{Conclusion}

We conclude that in case of non-PH, the choice of a suitable test procedure is relevant and the LR is not always the best choice. Therefore, we recommend to use all prior information available and to consider more options to test for differences in survival than just the LR. In terms of study design there are still some limitations since not all of the tests are used for sample size estimation and some tests are not freely available in $\mathrm{R}$ (see the Supplements for more information). Finally, we recommend using omnibus tests such as the mdir test for inference when no prior information on the pattern of hazards is available.

\section{Abbreviations}

2ST: Two-stage test; ABC: Area between curves; coxRMST: Combined Cox and permutation based RMST test; IPD: Individual patient data; KM: Kaplan-Meier; KONP chi: K-sample omnibus non-proportional hazards test with chi-square test statistic; KONP $\| r$ : K-sample omnibus non-proportional hazards test with log likelihood ratio type test statistic; LR: Log-rank test; mdir: Multiple-direction log-rank test; PH: Proportional hazards; PP: Peto-Peto test; RMST: Restricted mean suvival time.

\section{Supplementary Information}

The online version contains supplementary material available at https://doi. org/10.1186/s12874-022-01520-0.

Additional file 1. 


\section{Acknowledgements}

The authors are grateful to the editor, the associate editor and the two referees for their valuable feedback and suggestions that improved the quality of the paper.

\section{Authors' contributions}

All of the authors were involved in the planning of the study. Ina Dormuth conducted the literature review from which she searched, reconstructed and treated the data in R. This initial step was jointly supervised by Dr. Marc Ditzhaus and Prof. Dr. Markus Pauly. Dr. Tiantian Liu provided the R-Code for the ABC-method, which is not available as an R-package yet and participated in writing the methods section. Furthermore, Ina Dormuth prepared the first draft of the publication, which was then jointly polished by all authors. Prof. Dr. Jin Xu and Prof. Dr. Menggang Yu gave final notes for improvement. We followed the same procedure for the revision. The author(s) read and approved the final manuscript.

\section{Funding}

Open Access funding enabled and organized by Projekt DEAL. Marc Ditzhaus and Markus Pauly were supported by German Research Foundation Grant No PA 2409/5-1. The funders had no role in study design, data collection and analysis, decision to publish, or preparation of the manuscript.

\section{Availability of data and materials}

The datasets used and/or analyzed during the current study are available from the corresponding author on reasonable request.

\section{Declarations}

\section{Ethics approval and consent to participate}

We state that all methods were carried out in accordance with relevant guidelines and regulations.

\section{Consent for publication}

Not applicable.

\section{Competing interests}

The authors declare that they have no competing interests.

\section{Author details}

${ }^{1}$ TU Dortmund University, Joseph-von-Fraunhofer-Straße 2-4, 44221 Dortmund, Germany. ${ }^{2}$ Technion - Israel Institute of Technology, Haifa, Israel. ${ }^{3}$ East China Normal University, Shanghai, China. ${ }^{4}$ University of Wisconsin-Madison, Madison, USA.

Received: 24 June 2021 Accepted: 18 January 2022

Published online: 30 January 2022

\section{References}

1. Fleming TR, Lin DY. Survival analysis in clinical trials: past developments and future directions. Biometrics. 2000;56(4):971-83. https://doi.org/10. 1111/j.0006-341X.2000.0971.X.

2. Kleinbaum DG, Klein M. Survival Analysis, vol. 3: Springer; 2010.

3. Sharpe D. Why the resistance to statistical innovations? Bridging the communication gap. Psychol Methods. 2013;18(4):572-82. https://doi.org/10. 1037/a0034177.

4. Fleming TR, Harrington DP. Counting Processes and Survival Analysis. Wiley; 2011.

5. Kristiansen I. PRM39 survival curve convergences and crossing: a threat to validity of meta-analysis. Value Health. 2012;15(7):A652.

6. Ananthakrishnan R, Green S, Previtali A, Liu R, Li D, LaValley M. Critical review of oncology clinical trial design under non-proportional hazards. Crit Rev Oncol Hematol. 2021;162:103350. https://doi.org/10.1016/j.critr evonc.2021.103350

7. R Core Team. R: A Language and Environment for Statistical Computing. 2020
8. Royston P. A combined test for a generalized treatment effect in clinical trials with a time-to-event outcome. Stata J Promot Commun Stat Stata. 2017;17(2):405-21. https://doi.org/10.1177/1536867X1701700209.

9. Li H, Han D, Hou Y, Chen H, Chen Z. Statistical inference methods for two crossing survival curves: a comparison of methods. PLoS One. 2015;10(1):1-18.

10. Kraus D. Adaptive Neyman's smooth tests of homogeneity of two samples of survival data. J Stat Plan Inference. 2009;139(10):3559-69.

11. Qiu P, Sheng J. A two-stage procedure for comparing hazard rate functions. J R Stat Soc Ser B Stat Methodol. 2008;70(1):191-208.

12. Sheng J, Qiu P, Geyer CJ. TSHRC: Two Stage Hazard Rate Comparison. 2019. https://CRAN.R-project.org/package $=$ TSHRC. Accessed 25 Oct 2021.

13. Gorfine M, Schlesinger M, Hsu L. K-sample omnibus non-proportional hazards tests based on right-censored data. ArXiv Prepr ArXiv; 2019. p. 190105739.

14. Schlesinger M, Gorfine M. KONPsurv: KONP Tests: Powerful K-Sample Tests for Right-Censored Data.; 2020. https://CRAN.R-project.org/packa ge=KONPsurv. Accessed 25 Oct 2021

15. Yang S, Prentice R. Improved logrank-type tests for survival data using adaptive weights. Biometrics. 2010;66(1):30-8.

16. Brendel M, Janssen A, Mayer CD, Pauly M. Weighted Logrank permutation tests for randomly right censored life science data: weighted logrank permutation tests. Scand J Stat. 2014;41(3):742-61. https://doi.org/10. 1111/sjos.12059.

17. Ditzhaus M, Friedrich S. More powerful logrank permutation tests for two-sample survival data. ArXiv180705504 Math Stat. 2018. http://arxiv. org/abs/1807.05504. Accessed 6 May 2020

18. Ditzhaus M, Pauly M. Wild bootstrap logrank tests with broader power functions for testing superiority. Comput Stat Data Anal. 2019;136:1-11.

19. Ditzhaus M, Friedrich S. Mdir.Logrank: Multiple-Direction Logrank Test:; 2018. https://CRAN.R-project.org/package=mdir.logrank. Accessed 25 Oct 2021

20. Lee $\mathrm{SH}$. On the versatility of the combination of the weighted log-rank statistics. Comput Stat Data Anal. 2007:51(12):6557-64.

21. Wang Y, Wu H, Anderson KM, Roychoudhury S, Hu T, Liu H. NPHSIM: simulation and power calculations for time-to-event clinical trials; $2017 . \mathrm{R}$ package version 0.1.1.9000.

22. Kim DH, Uno H, Wei LJ. Restricted mean survival time as a measure to interpret clinical trial results. JAMA Cardiol. 2017;2(11):1179-80.

23. Tian L, Fu H, Ruberg SJ, Uno H, Wei LJ. Efficiency of two sample tests via the restricted mean survival time for analyzing event time observations: efficiency of two sample tests via the restricted mean survival time. Biometrics. 2018;74(2):694-702. https://doi.org/10.1111/biom.12770.

24. Uno H, Claggett B, Tian $L$, et al. Moving beyond the Hazard ratio in quantifying the between-group difference in survival analysis. J Clin Oncol. 2014;32(22):2380-5. https://doi.org/10.1200/JCO.2014.55.2208.

25. Tian L, Uno H, Horiguchi M. Surv2sampleComp: Inference for Model-Free Between-Group Parameters for Censored Survival Data. https://rdrr.io/ cran/surv2sampleComp/man/surv2sample.html. Accessed 25 Oct 2021.

26. Uno H, Tian L, Horiguchi M, Cronin A, Battioui C, Bell J. SurvRM2: Comparing Restricted Mean Survival Time; 2020. https://CRAN.R-project.org/ package=survRM2. Accessed 25 Oct 2021.

27. Royston P, Parmar MKB. Augmenting the logrank test in the design of clinical trials in which non-proportional hazards of the treatment effect may be anticipated. BMC Med Res Methodol. 2016;16(1):16. https://doi. org/10.1186/s12874-016-0110-x.

28. Royston P, Parmar MK. A simulation study comparing the power of nine tests of the treatment effect in randomized controlled trials with a time-to-event outcome. Trials. 2020;21(1):1-17. https://doi.org/10.1186/ s13063-020-4153-2.

29. Liu T, Ditzhaus M, Xu J. A resampling-based test for two crossing survival curves. Pharm Stat. 2020;19(4):399-409.

30. Guyot P, Ades A, Ouwens MJ, Welton NJ. Enhanced secondary analysis of survival data: reconstructing the data from published Kaplan-Meier survival curves. BMC Med Res Methodol. 2012;12(1):9. https://doi.org/10. 1186/1471-2288-12-9.

31. WebPlotDigitizer - Extract data from plots, images, and maps. https:// automeris.io/WebPlotDigitizer/. Accessed Oct 252021 
32. Matabuena M, Padilla OHM. Energy distance and kernel mean embeddings for two-sample survival testing. arXiv preprint arXiv:1912.04160, 2019.

33. Jones RL, Demetri GD, Schuetze SM, et al. Efficacy and tolerability of trabectedin in elderly patients with sarcoma: subgroup analysis from a phase III, randomized controlled study of trabectedin or dacarbazine in patients with advanced liposarcoma or leiomyosarcoma. Ann Oncol. 2018;29(9):1995-2002. https://doi.org/10.1093/annonc/mdy253.

34. Jones $\mathrm{RH}$, Casbard A, Carucci M, et al. Fulvestrant plus capivasertib versus placebo after relapse or progression on an aromatase inhibitor in metastatic, oestrogen receptor-positive breast cancer (FAKTION): a multicentre, randomised, controlled, phase 2 trial. Lancet Oncol. 2020;21(3):345-57. https://doi.org/10.1016/S1470-2045(19)30817-4.

35. Hammel $\mathrm{P}$, Kindler HL, Reni M, et al. Health-related quality of life in patients with a germline BRCA mutation and metastatic pancreatic cancer receiving maintenance olaparib. Ann Oncol. 2019;30(12):1959-68. https://doi.org/10.1093/annonc/mdz406.

36. Godfrey AL, Campbell PJ, MacLean C, et al. Hydroxycarbamide plus aspirin versus aspirin alone in patients with essential Thrombocythemia age 40 to 59 years without high-risk features. J Clin Oncol. 2018;36(34):3361-9. https://doi.org/10.1200/JCO.2018.78.8414.

37. Bang Y, Li C, Lee K, et al. Liposomal irinotecan in metastatic pancreatic adenocarcinoma in Asian patients: subgroup analysis of the NAPOLI-1 study. Cancer Sci. 2020;111(2):513-27. https://doi.org/10.1111/cas.14264.

38. Golan T, Hammel P, Reni M, et al. Maintenance Olaparib for germline BRCA -mutated metastatic pancreatic Cancer. N Engl J Med. 2019;381(4):31727. https://doi.org/10.1056/NEJMoa1903387.

39. Becker H, Pfeifer D, lhorst G, et al. Monosomal karyotype and chromosome 17p loss or TP53 mutations in decitabine-treated patients with acute myeloid leukemia. Ann Hematol. 2020;99(7):1551-60. https://doi. org/10.1007/s00277-020-04082-7.

40. Lu S, Chen Z, Hu C, et al. Nedaplatin plus docetaxel versus cisplatin plus docetaxel as first-line chemotherapy for advanced squamous cell carcinoma of the lung - a multicenter, open-label, randomized, Phase III Trial. J Thorac Oncol. 2018;13(11):1743-9. https://doi.org/10.1016/j.jtho.2018. 07.006 .

41. Ferris RL, Blumenschein G, Fayette J, et al. Nivolumab for recurrent squamous-cell carcinoma of the head and neck. N Engl J Med. 2016;375(19):1856-67. https://doi.org/10.1056/NEJMoa1602252.

42. Motzer RJ, Escudier B, McDermott DF, et al. Nivolumab versus Everolimus in advanced renal-cell carcinoma. N Engl J Med. 2015;373(19):1803-13. https://doi.org/10.1056/NEJMoa1510665.

43. Toxopeus E, van der Schaaf M, van Lanschot J, et al. Outcome of patients treated within and outside a randomized clinical trial on neoadjuvant Chemoradiotherapy plus surgery for esophageal Cancer: extrapolation of a randomized clinical trial (CROSS). Ann Surg Oncol. 2018;25(8):2441-8. https://doi.org/10.1245/s10434-018-6554-y.

44. Mukai H, Shimizu C, Masuda N, et al. Palbociclib in combination with letrozole in patients with estrogen receptor-positive, human epidermal growth factor receptor 2-negative advanced breast cancer: PALOMA-2 subgroup analysis of Japanese patients. Int J Clin Oncol. 2019;24(3):27487. https://doi.org/10.1007/s10147-018-1353-9.

45. Bellmunt J, de Wit R, Vaughn DJ, et al. Pembrolizumab as secondline therapy for advanced urothelial carcinoma. N Engl J Med. 2017;376(11):1015-26. https://doi.org/10.1056/NEJMoa1613683.

46. Cortes JE, Heidel FH, Hellmann A, et al. Randomized comparison of low dose cytarabine with or without glasdegib in patients with newly diagnosed acute myeloid leukemia or high-risk myelodysplastic syndrome. Leukemia. 2019;33(2):379-89. https://doi.org/10.1038/ s41375-018-0312-9.

47. Fradet Y, Bellmunt J, Vaughn DJ, et al. Randomized phase III KEYNOTE-045 trial of pembrolizumab versus paclitaxel, docetaxel, or vinflunine in recurrent advanced urothelial cancer: results of $>2$ years of follow-up. Ann Oncol. 2019;30(6):970-6. https://doi.org/10.1093/annonc/mdz127.

48. Kotani D. Retrospective cohort study of trifluridine/tipiracil (TAS-102) plus bevacizumab versus trifluridine/tipiracil monotherapy for metastatic colorectal cancer, vol. 9; 2019.

49. Malone S, Roy S, Eapen $L$, et al. Sequencing of androgen-deprivation therapy with external-beam radiotherapy in localized prostate Cancer: a phase III randomized controlled trial. J Clin Oncol. 2020;38(6):593-601. https://doi.org/10.1200/JCO.19.01904.
50. Kreuzer KA, Furman RR, Stilgenbauer S, et al. The impact of complex karyotype on the overall survival of patients with relapsed chronic lymphocytic leukemia treated with idelalisib plus rituximab. Leukemia. 2020;34(1):296-300. https://doi.org/10.1038/s41375-019-0533-6.

51. Tian $L$, Jin $H$, Uno $H$, et al. On the empirical choice of the time window for restricted mean survival time. Biometrics. 2020;76(4):1157-66. https://doi. org/10.1111/biom.13237.

52. Legrand C. Advanced survival models: CRC Press; 2021.

53. Trinquart L, Jacot J, Conner SC, Porcher R. Comparison of treatment effects measured by the Hazard ratio and by the ratio of restricted mean survival times in oncology randomized controlled trials. J Clin Oncol. 2016;34(15):1813-9. https://doi.org/10.1200/JCO.2015.64.2488.

54. Royston P. Combined test versus logrank/Cox test in 50 randomised trials. Trials. 2019;10:1-10.

55. Chen Z, Huang H, Qiu P. Comparison of multiple hazard rate functions. Biometrics. 2016;72(1):39-45.

56. Ditzhaus M, Genuneit J, Janssen A, Pauly M. CASANOVA: Permutation inference in factorial survival designs. Biometrics. 2021;1-13.

57. Chen Z, Huang H, Qiu P. An improved two-stage procedure to compare hazard curves. J Stat Comput Simul. 2017;87(9):1877-86.

\section{Publisher's Note}

Springer Nature remains neutral with regard to jurisdictional claims in published maps and institutional affiliations.
Ready to submit your research? Choose BMC and benefit from:

- fast, convenient online submission

- thorough peer review by experienced researchers in your field

- rapid publication on acceptance

- support for research data, including large and complex data types

- gold Open Access which fosters wider collaboration and increased citations

- maximum visibility for your research: over $100 \mathrm{M}$ website views per year

At BMC, research is always in progress.

Learn more biomedcentral.com/submissions 